\title{
Clinical Characteristics and Prevention Strategies of Falls in Hospitalized Patients with Chronic Kidney Disease
}

\author{
Chang Yin', Yumei Liao \\ ${ }^{1}$ Department of Nursing, Medical School of Yangtze University, Jingzhou, China \\ ${ }^{2}$ Department of Nephrology, Peking University Shenzhen Hospital, Shenzhen, China \\ Email: 1642673110@qq.com
}

How to cite this paper: Yin, C. and Liao, Y.M. (2020) Clinical Characteristics and Prevention Strategies of Falls in Hospitalized Patients with Chronic Kidney Disease. Yangtze Medicine, 4, 114-124.

https://doi.org/10.4236/ym.2020.42011

Received: July 20, 2019

Accepted: June 26, 2020

Published: June 29, 2020

Copyright $\odot 2020$ by author(s) and Scientific Research Publishing Inc. This work is licensed under the Creative Commons Attribution International License (CC BY 4.0).

http://creativecommons.org/licenses/by/4.0/

\begin{abstract}
Objectives: To analyze the clinical features of falls in hospitalized patients with chronic kidney disease (CKD), and to provide basis for preventing the occurrence of falls in hospitalized patients with CKD. Method: A retrospective study was conducted to analyze the 27 adverse events of falls in hospitalized patients from the department of nephrology in Peking University Shenzhen Hospital during May 2009 and May 2019. Results: Among the 27 patients with falls, people over 65 years old accounted for $55.56 \%$; $74.07 \%$ of the patients had caregivers; the peak period of falls was from June to August, accounting for $40.74 \%$, mainly occurring from 7:00 to 8:00, 12:00 to 13:30 and 19:00 to 21:00; the locations of falls were mostly near the bed and in the bathroom, accounting for $33.33 \%$ and $22.22 \%$, respectively; suffering from anemia, hypoproteinemia, abnormal serum potassium, calcium-phosphorus metabolism disorders accompanied by abnormal parathyroid hormone and hypertension accounted for $88.89 \%, 85.19 \%, 29.63 \%, 96.30 \%$ and $81.48 \%$ respectively. The inpatients on dialysis therapy accounted for $66.67 \%$. Conclusion: The occurrence of falls in hospitalized patients with CKD was not only related to age, caregivers, time, months, locations or other conventional factors, but also the complications and dialysis therapies. To effectively reduce the occurrence of falls, it's necessary to enhance the sense of safety and responsibility of caregivers. Besides, nurses should strengthen the inspection of wards for inpatients engaging in some intensive activities, and nurses should also pay more attention to the inpatients with anemia, hypoproteinemia, calcium-phosphorus metabolism disorders and those on dialysis therapies.
\end{abstract}

\section{Keywords}

Chronic Kidney Disease (CKD), Falls, Clinical Characteristics, Prevention Strategies 


\section{Introduction}

CKD has become a global public health problem due to its insidious onset, high prevalence, low awareness rate, high cost of treatment and poor prognosis [1]. In recent years, the prevalence of CKD in the general population of the world has reached $14.3 \%$, and in China, the prevalence of CKD in the population over the age of 18 has reached $10.8 \%$ [1]. Studies have shown that most patients with CKD have one or more complications, such as anemia, hypoproteinemia, cardiovascular diseases, bone mineral and calcium-phosphorus metabolism disorders and receive dialysis treatment, to some extent, these will increase the risk of falls in hospitalized patients with CKD [2] [3]. A fall is a sudden or unintended stop that the victim falls to the ground or lower position than the original [4]. According to statistics from the World Health Organization (WHO), 5\% - 15\% of falls would result in the injuries, such as brain damages, soft tissue contusions, fractures or dislocations and so on [5]. During hospitalization, once a patient falls, it will not only increase the patient's pain, aggravate the state of illness, prolong the length of hospitalization, but also further increase the economic burden of the patient, consume additional medical resources, induce medical disputes and cause conflicts between nurses and patients [6]. Therefore, preventing and reducing the occurrence of falls in hospitalized patients have become one of the goals of hospital safety management. The purposes of this paper were to explore the clinical features of falls in hospitalized patients with CKD and summarize the relevant prevention measures.

\section{Materials and Methods}

\subsection{Study Sample}

The study sample included 27 adverse events of falls for inpatients from the department of nephrology in Peking University Shenzhen Hospital from May 2009 to May 2019.

\subsubsection{Inclusion Criteria}

1) The objects meet the hospital's definition of adverse events related to falls. The adverse events of falls include all kinds of sudden or unintended stops that the patients fall to the ground or lower position than the original [4], for example, falls while walking, falls by the bed or chair and so on. 2) The information and data of related adverse events should be completed, including general information of the patients (such as gender, age, primary diseases, hypertention or not, dialysis modalities and with or without caregiver); laboratory examination indexes (such as hemoglobin, albumin, serum potassium, serum calcium, serum phosphorus and parathyroid hormone) and the details of falls (such as time, months, locations, causes and outcomes).

\subsubsection{Exclusion Criteria}

The related adverse events of falls had disputes. 


\subsection{Methods}

\subsubsection{Data Collection}

To refer to the reports and corresponding electronic medical records of adverse events of falls in the department of nephrology of Peking University Shenzhen Hospital from May 2009 to May 2019. To record general information of the patients (such as gender, age, primary diseases, hypertention or not, dialysis modalities and with or without caregiver); laboratory examination indexes (such as hemoglobin, albumin, serum potassium, serum calcium, serum phosphorus and parathyroid hormone) and the details of falls (such as time, months, locations, causes and outcomes).

\subsubsection{Statistical Methods}

The selected data should be confirmed by two individuals, the researchers downloaded the original data to Excel files and then analyzed the relevant data by importing them into SPSS19.0 software. The quantitative data was expressed as mean \pm standard deviation, the count data was described by frequency and rate.

\subsection{Index Definitions}

The diagnostic criteria for renal anemia are recommended by WHO: area with sea-level, $\geq 15$ years old, the level of hemoglobin of men is under $130 \mathrm{~g} / \mathrm{L}$ or the level of hemoglobin of adult non-pregnant female is under $120 \mathrm{~g} / \mathrm{L}$ [7]. Hypoproteinemia is defined that the level of albumin is under $35 \mathrm{~g} / \mathrm{L}$ [8]. The level of serum potassium, serumcalcium, serum phosphorus and parathyroid hormone are referred to the normal range of laboratory examination indexes in Peking University Shenzhen Hospital, which are $3.5-5.3 \mathrm{mmol} / \mathrm{L}, 2.11-2.52 \mathrm{mmol} / \mathrm{L}$, $0.85-1.51 \mathrm{mmol} / \mathrm{L}$ and $0-6.5 \mathrm{pmol} / \mathrm{L}$ respectively.

\section{Results}

\subsection{General Information on the Hospitalized Patients with Falls}

The average age of the 27 patients with falls was $(63.19 \pm 13.11)$ years old, the youngest one of them was 33 years old and the oldest one was 91 years old. The primary diseases of these patients included: chronic glomerulonephritis (9/27), nephrotic syndrome (10/27), diabetic nephropathy (5/27), hypertensive nephropathy combined with diabetic nephropathy (1/27), gout nephropathy $(1 / 27)$ and IgA nephropathy (1/27). Additionally, the complications of the 27 patients were anemia $(88.89 \%, 24 / 27)$, hypoproteinemia $(85.19 \%, 23 / 27)$, hypokalemia $(25.93 \%, 7 / 27)$, Hyperkalemia $(3.70 \%, 1 / 27)$ and calcium phosphorus metabolism disorder accompanied with parathyroid hormone abnormality $(96.30 \%, 26 / 27)$ respectively.

\subsection{Information on the Falls}

The three high-risk periods of falls were respectively 7:00-8:00 (6/27), 12:00-13:30 
(6/27) and 19:00-21:00 (6/27). The main causes of falls during the first period were falling beside the bed when patients got up to sit on the chair in the morning (2/27), falling while washing or toileting (2/27) and falling while walking after breakfast (2/27). The major causes of falls during the last two periods were falling while doing some activities outside the hospital (3/27), falling on the way to bathroom (1/27), falling when patients got up after toileting (4/27), falling when the patient stepped on the chair to take some goods (1/27), falling while walking in the hallway of the inpatient area $(2 / 27)$ and falling when the patient stood up after getting up from the bed suddenly (1/27). The causes of falls during other periods were falling while doing some activities without the help of caregivers (4/27), falling after the patient fell asleep while sitting on the chair $(1 / 27)$, falling after the caregivers left without putting patients in correct positions (2/27) and falling due to some diseases (2/27).

The adverse events related to falls include skin abrasions, pain and swelling in joints, fractures, dizziness, nausea and loss of consciousness. With the timely detection and effective care of nurses, the symptoms related to falls in the 27 hospitalized patients with CKD have improved.

\section{Discussion}

\subsection{The Analysis of Common Factors and Prevention Strategies of Falls in CKD Patients}

\subsubsection{Demographic Factors}

According to Table 1 , there were slightly more males $(51.85 \%, 14 / 27)$ than females $(48.15 \%, 13 / 27)$ in the 27 patients of falls, which was consistent with the result of Jiang's [2]. Patients over 65 years old accounted for 55.56\% (15/27), thus it could be found that the elderly were at high risk of falls. According to the

Table 1. General information on the hospitalized patients with falls $(n=27)$.

\begin{tabular}{|c|c|c|c|}
\hline Items & Options & Frequency & Rate (\%) \\
\hline \multirow{2}{*}{ Gender } & Male & 14 & 51.85 \\
\hline & Female & 13 & 48.15 \\
\hline \multirow{3}{*}{ Age, years } & $<65$ & 12 & 44.44 \\
\hline & $65-70$ & 10 & 37.04 \\
\hline & $>70$ & 5 & 18.52 \\
\hline \multirow{2}{*}{ Hypertention } & Yes & 22 & 81.48 \\
\hline & No & 5 & 18.52 \\
\hline \multirow{3}{*}{ Dialysis modalities } & Hemodialysis (HD) & 8 & 29.63 \\
\hline & Peritoneal Dialysis (PD) & 10 & 37.04 \\
\hline & No & 9 & 33.33 \\
\hline \multirow{2}{*}{ Caregiver } & Yes & 20 & 74.07 \\
\hline & No & 7 & 25.93 \\
\hline
\end{tabular}


relevant researches, as to the elderly, falls have become the leading cause of death and an important factor of disabilities [9]. The severe deterioration of physical functions, abnormal gait cycles, reduced mobility and visual or auditory impairment; suffering from one or more diseases, such as hypertention or stroke; together with the general psychological resistance to aging, reluctance to bother others and fear of falling, all of these will cause the elderly to lose balance or stability of themselves and fail to take adequate precautions, thereby increasing the risk of falls [10] [11] [12] [13]. Guo's study further pointed that among the elderly patients, the self-efficacy of falls of female was lower than that of male [14]. Therefore, nurses should strengthen the health education of inpatients (especially for the elderly), solve the problems of disobedience to aging and unwillingness of bothering others, help female patients establish the confidence to prevent falls, prevent male patients from falling due to the blind confidence [10] and guide the caregivers to accompany patients (especially for the elderly) to have meals, go to toilets, wash or take showers, go for walks or do other activities, especially avoid the elderly patients doing activities alone.

\subsubsection{Factors in Caregiver}

According to Table 1, 74.07\% (20/27) of the 27 patients with falls had caregivers and among all the reasons of falls, $7.41 \%$ (2/27) of the patients fell caused by the negligence of caregivers. It was mainly related to the weak safety consciousness and lack of knowledge of prevention measures of caregivers. Then it was also related to the overwork and fatigue of caregivers due to the long-term care for patients. Therefore, nurses should inform patients who at high risk of falls and their families to leave a caregiver to accompany the patient during hospitalization, tell caregivers the risk factors of falls and prevention measures, suggest caregivers to take care of patients by other staffs in turn when they are exhausted and the handover work should be done well between different staffs.

\subsubsection{Time and Months of Falls}

According to Figure 1, there were three high-risk periods of falls in hospitalized patients with CKD, which were 7:00-8:00 (6/27), 12:00-13:30 (6/27) and 19:00-21:00 (6/27) respectively. The occurrence of falls from 7:00 to 8:00 was related to the fact that the patients' unconsciousness when they woke up, the caregivers' negligence and the nurses' negligence in inspecting the wards for being busy with blood collection or other works [15]. The occurrence of falls from 12:00 to $13: 30$ and 19:00 to 21:00 were related to the fact that the patients' activities such as having meals, washing or going to toilets were relatively intensive, the patients left the hospital without obeying regulations, the patients' awareness of preventing falls was weak and the nurses' health education in patients was inadequate [2] [15]. Therefore, nurses should emphasize repeatedly that leaving hospital is not permitted during hospitalization; explain relevant knowledge of preventing falls in detail, such as no stamping on tables, chairs, beds or other items to take some goods, and the importance of "Three Slow" principle: lie in 


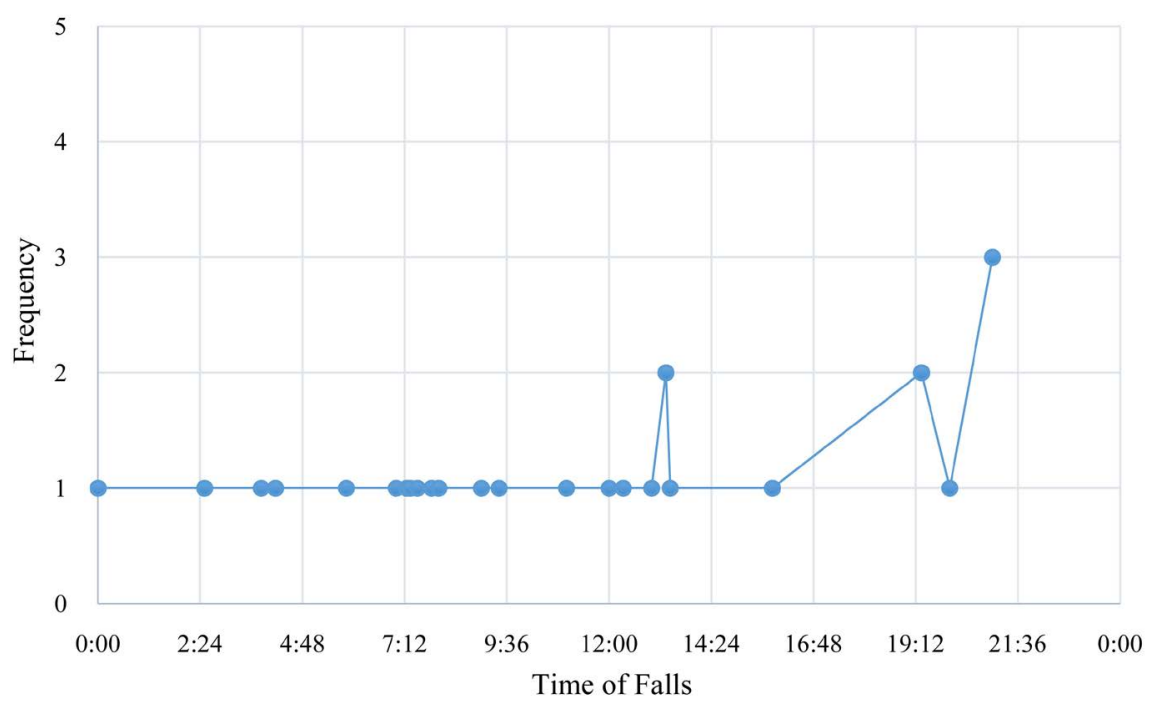

Figure 1 . Time of falls $(n=27)$.

bed for 30 seconds after waking up, sit up slowly, stand up slowly after sitting for 30 seconds, then walk slowly after standing up for 30 seconds. In addition, nurses should strengthen the inspection of wards, further improve the awareness of precautions, regularly assess the risk level of falls, ask patients questions about the knowledge of related prevention measures and offer regular education to the patients with risk of falls. According to Figure 2, the peak period of falls in hospitalized patients with CKD was from June to August, accounting for $40.74 \%$ (11/27), and the second period was from March to May, accounting for $33.33 \%$ $(9 / 27)$. The hospital selected in this study is located in Shenzhen, where the climate is mild and humid, and the rainfall is abundant [16]. According to Cai's study, in recent years, the rainy period in Shenzhen was mainly from April to September, and the most abundant precipitation was from June to August, additionally, during this period the weather in Shenzhen was moist and the ground got damp severely, all of these would increase the risk of falls [16]. Wu's study further indicated that March was the transition period of winter and spring, the temperature during this period changed greatly, which increased the incidence of cardiovascular and cerebrovascular diseases, and these diseases or some drugs such as hypotensive drugs would further increase the risk of falls by affecting patients' blood pressure, consciousness and balance ability [17]. Therefore, firstly, nurses should regularly tell patients to pay attention to the slippery ground, especially avoid doing some activities on the slippery floor. Secondly, nurses should help the cleaning staffs to keep the floor clean and dry, place warning signs to remind patients when the floor is slippery. Thirdly, nurses should observe the patients' condition closely and instruct them not to move immediately after taking medicine.

\subsubsection{Locations of Falls}

As were shown in Table 2, falls in hospitalized patients with CKD occurred mostly beside the bed, accounting for $33.33 \%$ (9/27). The second location was in 


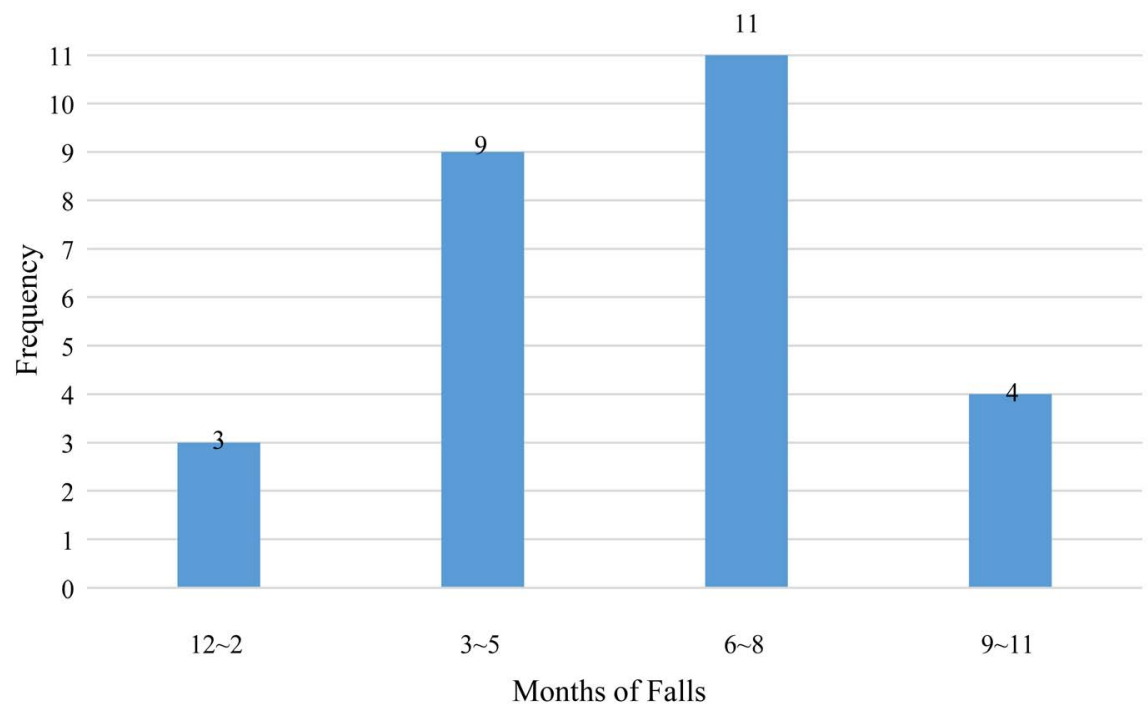

Figure 2. Months of falls $(n=27)$.

Table 2. Locations of falls $(n=27)$.

\begin{tabular}{cccc}
\hline Items & Options & Frequency & Rate (\%) \\
\hline Beside the bed & 9 & 33.33 \\
In the bathroom & 6 & 22.22 \\
Locations & In the ward & 3 & 11.11 \\
& In the hallway of the inpatient area & 4 & 14.81 \\
Outside the hospital & 5 & 18.52 \\
\hline
\end{tabular}

the bathroom, accounting for $22.22 \%$ (6/27). The proportion of falls occurred outside the hospital due to outdoor activities was $18.52 \%$ (5/27). This was basically consistent with the result of Jiang's study [2]. These were mainly related to the patients getting out of bed without the caregivers' knowledge, sudden changes of body positions, sudden discomforts in the process of washing or toileting and leaving hospital without permissions. Therefore, "Three Slow" principle and "patients during hospitalization are strictly forbidden to go out" should be emphasized by nurses patiently to the patients and their caregivers. Additionally, nurses should also illustrate the severe consequences of postural hypotension caused by sudden changes of postural positions, tell caregivers pay more attention to the patients, especially for the elderly and avoid them acting alone, suggest patients to wear non-slip shoes or slippers. If the patients feel unwell, their washing and defecation should be done on the bed with the help of caregivers and avoid getting out of bed. Besides, non-slip handrails should be installed in the corridors of bathrooms and wards, all kinds of sundries should be cleared in time, keep the floor of the wards (especially the bathrooms) clean, dry and free of obstacles, and turn on the floor lamp at night. 


\subsection{The Analysis of Specific Causes and Prevention Measures of Falls in CKD Patients}

\subsubsection{Causes of Complications}

Firstly, according to the results of this study, there were $88.89 \%(24 / 27)$ of the patients with CKD accompanying with anemia. Wang's study indicated that anemia was the common complication of patients with CKD, regardless of gender, the incidence of anemia of them would increase with age, especially for the patients with CKD over 70 years old, the incidence of anemia of them was more than $80 \%$ [18]. According to Jiang's study, anemia would cause the insufficient of oxygen supply which could further lead to abnormal clinical features such as lacking in strength, dizziness and other symptoms, these may lead to the falls in patients with CKD [2]. Secondly, in this study, there were $85.19 \%$ (23/27) of the patients with CKD with hypoproteinemia, and it is well known that the level of albumin is one of the serological indexes to evaluate nutritional status. According to Guo's study, there was a higher incidence of falls for those who were malnutrition [19]. So clinical staffs should pay more attention to the levels of hemoglobin and albumin of patients with CKD during their hospitalization, and give them some suitable suggestions of diet to improve their health. Thirdly, among the 27 patients with falls, there were $25.93 \%$ (7/27) of the patients with CKD accompanying with hypokalemia and 3.7\% (1/27) accompanying with hyperkalemia. Hypokalemia is likely to cause patients with dizziness, limbs weakness and other discomforts, while hyperkalemia is likely to cause patients with limbs numbness, muscle pain and arrhythmia, both of the two conditions would increase the risk of falls. Therefore, in order to control the level of serum potassium, nurses should pay more attention to the variation of serum potassium of patients, give them some suggestions of rational use of potassium supplement or potassium lowering drugs and help them distinguish food between high potassium and low potassium. Fourthly, the patients who had calcium-phosphorus metabolism disorders accompanying by abnormal parathyroid hormone accounted for $96.30 \%$ (26/27). Foreign and domestic studies documented that patients with CKD had a high incidence of calcium-phosphorus metabolism disorders, and long-term calcium-phosphorus metabolism disorders could lead to hyperparathyroidism, mineral and bone metabolism abnormalities, which would lead to renal bone disease, result in osteoporosis, joint pain and limited movement, and further increase the risk of falls [2] [20] [21]. Therefore, nurses should explain the methods of serum calcium supplementation and serum phosphorus reduction to the patients and their caregivers in detail, guide them to take serum calcium tablets or other drugs correctly, monitor the level of serum calcium, serum phosphorus and parathyroid hormone regularly, communicate with patients and doctors immediately if any abnormality happens. Finally, the patients accompanying with hypertension accounted for $81.48 \%$ (22/27). Wu's study indicated that cardiovascular diseases such as hypertension may lead to dizziness and physical fatigue in patients, thereby increasing the risk of falls [17]. So nurses should guide patients to take antihypertensive drugs correctly, 
tell them that immediate activities are strictly forbidden after taking these drugs and blood pressure should be monitored regularly during their hospitalization.

\subsubsection{Causes of Therapies}

$\mathrm{HD}$ and PD are two main renal replacement therapies for patients with CKD. As the result shown in Table 1, among the 27 patients with falls in this study, $29.63 \%(8 / 27)$ of them were treated with HD and $37.04 \%$ (10/27) of them were treated with PD. According to previous studies, patients undergoing HD had a high risk of falls, it mainly occurred during the process of dialysis or on the way home after dialysis, and falls would occur more frequently if there were no caregivers with them [22] [23]. The falls in patients undergoing HD may attribute to dialysis-related hypotension, dialysis-related arrhythmia, insufficient exercise tolerance, post-dialysis fatigue and unqualified environment of dialysis center or other factors [22] [24]. So nurses should regularly monitor the blood pressure of patients during dialysis, tell them to take blood pressure at the end of dialysis and they could go back to wards only when the blood pressure is normal. As for the patients at high risk of falls, a caregiver is recommended during and after their dialysis. While Wen's study indicated that patients undergoing PD had a higher risk of osteoporosis and falls than ordinary patients, and it was due to abnormal metabolism [25]. Therefore, nurses should guide patients to take vitamin D supplements properly to keep it at a normal level, which can effectively prevent osteoporosis and reduce the occurrence of falls [26].

\section{Conclusion}

A retrospective study was conducted to analyze the clinical features of falls in 27 hospitalized patients with CKD. Our study summarized that the occurrence of falls in patients with CKD was not only related to age, caregivers, time, months, locations or other conventional factors but also the complications and dialysis therapies. In order to minimize the occurrence of falls and optimize the medical services, the sense of safety and responsibility of caregivers should be further strengthened, and nurses should strengthen the inspection of wards during inpatients having meals, washing, toileting or engaging in other intensive activities. Besides, nurses should also pay more attention to the inpatients with anemia, hypoproteinemia, calcium-phosphorus metabolism disorders and those on dialysis therapies.

\section{Limitation}

There were 27 cases in this study, the sample size was small. But we have made a detailed analysis of general information, laboratory examination indexes and relevant information on falls in the 27 hospitalized patients with CKD. Therefore, to some extent, our study could provide basis for preventing the occurrence of falls in hospitalized patients with CKD and giving some alerts to medical staffs. To be more scientific, the future investigation and analysis of falls in hospitalized patients with CKD can be conducted in multiple hospitals. 


\section{Conflicts of Interest}

The authors declare no conflicts of interest regarding the publication of this paper.

\section{References}

[1] Expert Group on Early Detection DATS (2017) Guideline for Screening, Diagnosis, Prevention and Treatment of Chronic Kidney Disease. Chinese Journal of Practical Internal Medicine, 37, 28-34.

[2] Jiang, W., Wen, Y., Diao, Y., Ni, Y., Chen, X. and Li, X. (2017) Analysis of Correlative Factors of Tumble for Inpatients with Chronic Kidney Disease and Countermeasures. Medical Journal of West China, 29, 1009-1013.

[3] Miller, E. (2015) Chronic Kidney Disease. Foster Academics, Princeton, NJ.

[4] Severo, I.M., et al. (2014) Risk Factors for Falls in Hospitalized Adult Patients: An Integrative Review. Revista da Escola de Enfermagem da USP, 48, 540-554. https://doi.org/10.1590/S0080-623420140000300021

[5] King, M.B. and Tinetti, M. (1996) A Muhffactorial Approach to Reducing Injurious Fall. Clinics in Geriatric Medicine, 12, 745-759. https://doi.org/10.1016/S0749-0690(18)30199-X

[6] Davis-Ajami, M.L., Fink, J.C. and Baernholdt, M. (2019) Adverse Safety Event Characteristics and Predictive Factors in Hospital Encounters for Patients with Chronic Kidney Disease. American Journal of Nephrology, 50, 72-80. https://doi.org/10.1159/000500562

[7] Wang, L. and Yuan, W. (2018) Review of the Guidelines and Consensus about the Treatment of Renal Anemia. Chinese Journal of Blood Purification, 17, 1-5.

[8] He, L., Zhang, H., Chang, L. and Lu, X. (2017) Analysis on Nutritional Status of Patients with Chronic Kidney Disease. Nursing and Rehabilitation Journal, 16, 225-229.

[9] Shijun, W. (2007) Gerontological Nursing. People's Military Medical Publisher, Beijing.

[10] Jing, L., Wei, L. and Du, H. (2017) Falls Self-Efficacy in the Elderly People. Modern Clinical Nursing, 16, 53-58.

[11] Shu, X., Shen, Z.-X., Zheng, H., et al. (2019) Assessment of Fall Risk and Related Risk Factors in the Elderly. Pract Geriatr, 33, 190-193.

[12] Scott, I.A., Hilmer, S.N. and Le Couteur, D.G. (2019) Going beyond the Guidelines in Individualising the Use of Antihypertensive Drugs in Older Patients. Drugs \& Aging, 36, 675-685. https://doi.org/10.1007/s40266-019-00683-8

[13] Tran, J., Ayers, E., Verghese, J., et al. (2019) Gait Abnormalities and the Risk of Falls in CKD. Clinical Journal of the American Society of Nephrology, 14, 983-993. https://doi.org/10.2215/CJN.13871118

[14] Guo, H., Li, H.-Y., Yang, Y.-W., et al. (2013) The Influencing Factors of Falls Efficacy among Hospitalized Older Patients with Cerebrovascular Disease. Chinese Journal of Nursing, 48, 147-150.

[15] Chen, Y., Zhang, G. and Li, S. (2019) A Retrospective Study of 168 Inpatients with Adverse Events Related to Falls. Shanghai Nursing, 19, 60-62.

[16] Weiyuan, C., Zhenzhen, C. and Zekai, P. (2017) Changes of Temperature and Precipitation in Shenzhen in Recent 63 Years. Guangdong Meteorology, 39, 6-9.

[17] Wu, T., Li, Y. and Yao, J. (2009) Investigation on Time Pattern of Unintentional 
Falls in Patients with Cardiovascular Diseases. Journal of Nursing Science, 24, 32-33. https://doi.org/10.7748/ns.24.4.11.s16

[18] Wang, Y. (2016) Investigation and Analysis of Status of Anemia in Patients with Chronic Kidney Disease. Hubei Journal of TCM, 38, 16-18.

[19] Guo, X., Zhao, L., Yang, M., et al. (2017) Corelation between the Risk of Falling and Nutritional Status in Elderly Patients. Chinese Journal of Clinical Healthcare, 20, 441-443.

[20] Labriola, L., Jadoul, M. and Cohen-Solal, M. (2018) Fractures in CKD Patients: Action Plans Should Not Overlook the Prevention of Falls. Kidney International, 93, 1247-1250. https://doi.org/10.1016/j.kint.2018.01.019

[21] Chen, H., Lips, P., Vervloet, M.G., et al. (2018) Association of Renal Function with Bone Mineral Density and Fracture Risk in the Longitudinal Aging Study Amsterdam. Osteoporosis International, 29, 2129-2138. https://doi.org/10.1007/s00198-018-4592-8

[22] Zhu, S. and Yuan, H. (2016) Causes of Falls in Maintenance Hemodialysis Patients and the Specialization Preventive Measures. Nursing Practice and Research, 13, 34-35.

[23] Plantinga, L.C., Patzer, R.E., Franch, H.A., et al. (2017) Serious Fall Injuries Before and After Initiation of Hemodialysis among Older ESRD Patients in the United States: A Retrospective Cohort Study. American Journal of Kidney Diseases, 70, 76-83. https://doi.org/10.1053/j.ajkd.2016.11.021

[24] Zanotto, T., Mercer, T. van der Linden, M.L., et al. (2018) Baroreflex Function, Haemodynamic Responses to an Orthostatic Challenge, and Falls in Haemodialysis Patients. PLoS ONE, 13, e208127. https://doi.org/10.1371/journal.pone.0208127

[25] Wen, L., Zhang, H., Wang, L. and Jiang, Z. (2017) Effects of Vitamin D Supplementation on Peritoneal Dialysis Patients. Nursing Practice and Research, 14, $39-40$.

[26] Demircioglu, D.T. (2018) The Association of Vitamin D Levels and the Frailty Phenotype among Non-Geriatric Dialysis Patients: A Cross-Sectional Study. Clinics, 73, 1-5. https://doi.org/10.6061/clinics/2018/e116 